\title{
Class of Analytic Function Related with Uniformly Convex and Janowski's Functions
}

\author{
Akhter Rasheed $\mathbb{D}^{1},{ }^{1}$ Saqib Hussain $\mathbb{D}{ }^{2}$ Muhammad Asad Zaighum, ${ }^{1}$ and Maslina Darus $\mathbb{D D}^{3}$ \\ ${ }^{1}$ Department of Mathematics and Statistics, Riphah International University, Islamabad, Pakistan \\ ${ }^{2}$ Department of Mathematics, COMSATS University Islamabad, Abbottabad Campus, Pakistan \\ ${ }^{3}$ Faculty of Science and Technology, Universiti Kebangsaan Malaysia, Bangi 43600, Selangor, Malaysia
}

Correspondence should be addressed to Maslina Darus; maslina@ukm.edu.my

Received 21 July 2018; Accepted 18 September 2018; Published 16 October 2018

Academic Editor: Seppo Hassi

Copyright @ 2018 Akhter Rasheed et al. This is an open access article distributed under the Creative Commons Attribution License, which permits unrestricted use, distribution, and reproduction in any medium, provided the original work is properly cited.

In this paper, we introduce a new subclass of analytic functions in open unit disc. We obtain coefficient estimates, extreme points, and distortion theorem. We also derived the radii of close-to-convexity and starlikeness for this class.

\section{Introduction}

Let $\mathscr{A}$ denote the class of normalized analytic functions in open unit disc $\Delta=\{z:|z|<1\}$ and having Taylor series of form

$$
f(z)=z+\sum_{n=2}^{\infty} a_{n} z^{n}
$$

Silverman [1] introduced and studied a subclass $\mathscr{A}^{-}$of $\mathscr{A}$ consisting of functions of the form

$$
f(z)=z-\sum_{n=2}^{\infty} a_{n} z^{n}, \quad a_{n}>0, z \in \Delta .
$$

A complex valued function $f(z)$ is said to be univalent in $\Delta$ if $z_{1} \neq z_{2}$ implies $f\left(z_{1}\right) \neq f\left(z_{2}\right)$, for all $z_{1}, z_{2} \in \Delta$. Let $\delta$ be the subclass of $\mathscr{A}$ composed of univalent functions in $\Delta$. By $\mathcal{S} \mathscr{T}, \mathscr{C} \mathscr{V}$, and $\mathscr{K}$, we mean the well-known subclasses of $\mathcal{S}$ that are starlike, convex, and close-to-convex functions, respectively; for detail see $[2,3]$.

In 1991, Goodman [2, 3] introduced classes $\mathscr{U} \mathscr{C V}$ and $\mathscr{U} \mathcal{S} \mathscr{T}$ of uniformly convex and uniformly starlike functions, respectively. A function $f \in \mathcal{S}$ is uniformly convex if $f(z)$ maps every circular arc $\gamma$ contained in $\Delta$ with center $\zeta \in \Delta$ onto a convex arc. The function $f \in \mathcal{S}$ is uniformly starlike if $f(z)$ maps every circular arc $\zeta$ contained in $\Delta$ with center $\zeta \in \Delta$ onto a starlike arc with respect to $f(\zeta)$. A more useful representation of $\mathcal{U} \mathscr{C} \mathscr{V}$ and $\mathcal{U} \mathcal{S} \mathscr{T}$ was given in [2, 3]; see also [4-7] as

$$
\begin{aligned}
& f \in \mathcal{U} \mathscr{C} \mathscr{V} \Longleftrightarrow \\
& f \in \mathscr{A}
\end{aligned}
$$

$$
\text { and } \operatorname{Re}\left(1+\frac{z f^{\prime \prime}(z)}{f^{\prime}(z)}\right)>\left|\frac{z f^{\prime \prime}(z)}{f^{\prime}(z)}\right|, \quad z \in \Delta,
$$

and

$$
\begin{aligned}
& f \in \mathcal{U} \mathcal{S} \mathscr{T} \Longleftrightarrow \\
& f \in \mathscr{A}
\end{aligned}
$$

$$
\text { and } \operatorname{Re}\left(\frac{z f^{\prime}(z)}{f(z)}\right)>\left|\frac{z f^{\prime}(z)}{f(z)}-1\right|, \quad z \in \Delta,
$$

In 2011, Noor et. al [8] introduced and studied a class $k-$ $\mathscr{U} \mathcal{S} \mathscr{T}(A, B), k \geq 0,-1 \leq B<A \leq 1$ as follows.

A function $f(z) \in \mathscr{A}$ is said to be in the class $k-$ $\mathcal{U} \mathcal{S} \mathscr{T}(A, B)$ if and only if

$$
\begin{aligned}
\operatorname{Re}\left[\frac{(B-1)\left(z f^{\prime}(z) / f(z)\right)-(A-1)}{(B+1)\left(z f^{\prime}(z) / f(z)\right)-(A+1)}\right] \\
\quad>k\left|\frac{(B-1)\left(z f^{\prime}(z) / f(z)\right)-(A-1)}{(B+1)\left(z f^{\prime}(z) / f(z)\right)-(A+1)}-1\right| .
\end{aligned}
$$


The abovementioned few classes were widely investigated by many authors in the last decades; see [4, 8-15] and the references cited therein. By taking motivation from cited work, we define a unified class of analytic functions as follows.

Definition 1. A function $f(z) \in \mathscr{A}$ is said to be in the class $k-\mathscr{U} \mathcal{S} \mathscr{T}(A, B, \delta, \lambda)$ if and only if

$$
\begin{aligned}
& \operatorname{Re}\left[\frac{(B-1)\left(\left(z f^{\prime}(z)+\delta\left((1+2 \lambda) z^{2} f^{\prime \prime}(z)+\lambda z^{3} f^{\prime \prime \prime}(z)\right)\right) /\left((1-\delta) f(z)+\delta\left(z f^{\prime}(z)+\lambda z^{2} f^{\prime \prime}(z)\right)\right)\right)-(A-1)}{(B+1)\left(\left(z f^{\prime}(z)+\delta\left((1+2 \lambda) z^{2} f^{\prime \prime}(z)+\lambda z^{3} f^{\prime \prime \prime}(z)\right)\right) /\left((1-\delta) f(z)+\delta\left(z f^{\prime}(z)+\lambda z^{2} f^{\prime \prime}(z)\right)\right)\right)-(A+1)}\right] \\
& >k \mid \frac{(B-1)\left(\left(z f^{\prime}(z)+\delta\left((1+2 \lambda) z^{2} f^{\prime \prime}(z)+\lambda z^{3} f^{\prime \prime \prime}(z)\right)\right) /\left((1-\delta) f(z)+\delta\left(z f^{\prime}(z)+\lambda z^{2} f^{\prime \prime}(z)\right)\right)\right)-(A-1)}{(B+1)\left(\left(z f^{\prime}(z)+\delta\left((1+2 \lambda) z^{2} f^{\prime \prime}(z)+\lambda z^{3} f^{\prime \prime \prime}(z)\right)\right) /\left((1-\delta) f(z)+\delta\left(z f^{\prime}(z)+\lambda z^{2} f^{\prime \prime}(z)\right)\right)\right)-(A+1)} \\
& \quad-1 \mid
\end{aligned}
$$

where $0 \leq \delta \leq 1,0 \leq \lambda<1, k \geq 0,-1 \leq B<A \leq 1$.

We further let $k-\mathscr{V} \mathscr{U} \mathcal{S} \mathscr{T}(A, B, \delta, \lambda)=k-$ $\mathcal{U} \mathscr{S}(A, B, \delta, \lambda) \cap \mathscr{A}^{-}$.

\section{Special Cases}

(i) $k-\mathscr{U} \mathcal{S} \mathscr{T}(A, B, 0, \lambda)=k-\mathscr{U} \mathcal{S} \mathscr{T}(A, B)[8]$.

(ii) $k-\mathscr{U} \mathcal{S} \mathscr{T}(A, B, 1,0)=k-\mathscr{U} \mathscr{C} \mathscr{V}(A, B)[8]$.

(iii) $k-\mathscr{U} \mathcal{S} \mathscr{T}(1-2 \alpha,-1,0, \lambda)=k-\mathscr{U} \mathcal{S} \mathscr{T}(\alpha)[16]$.

(iv) $k-\mathscr{U} \mathcal{S} \mathscr{T}(1-2 \alpha,-1,1,0)=k-\mathscr{U} \mathscr{C} \mathscr{V}(\alpha)[16]$.

(v) $k-\mathscr{U} \mathcal{S} \mathscr{T}(1,-1,0, \lambda)=k-\mathcal{U} \mathcal{S} \mathscr{T}[14]$.

Throughout this paper $0 \leq \delta \leq 1,0 \leq \lambda<1, k \geq 0$-, and $-1 \leq B<A \leq 1$, unless otherwise stated.
In the next section we shall present our major findings as theorems and corollaries.

\section{Main Results}

Theorem 2. Let $f \in \mathscr{A}$ be given by (1); then $f$ is in $k-$ $\mathcal{U S T}(A, B, \delta, \lambda)$ if

$$
\begin{aligned}
& \sum_{n=2}^{\infty}\left\{2(k+1)(n-1) \Phi_{n}+\left|(B+1) \Psi_{n}-(A+1) \Phi_{n}\right|\right\} \\
& \cdot\left|a_{n}\right| \leq|B-A|,
\end{aligned}
$$

where

$$
\Phi_{n}=(1-\delta)+\delta n(1+\lambda(n-1))
$$

$$
\text { and } \Psi_{n}=(n+\delta n(n-1)(1+\lambda n)) \text {. }
$$

Proof. Assume that inequality (7) holds true. Then we have

$$
\begin{aligned}
k \mid & \frac{(B-1)\left(\left((1-\delta) z f^{\prime}+\delta\left(z f^{\prime}+(1+2 \lambda) z^{2} f^{\prime \prime}+\lambda z^{3} f^{\prime \prime \prime}\right)\right) /\left((1-\delta) f+\delta\left(z f^{\prime}+\lambda z^{2} f^{\prime \prime}\right)\right)\right)-(A-1)}{(B+1)\left(\left((1-\delta) z f^{\prime}+\delta\left(z f^{\prime}+(1+2 \lambda) z^{2} f^{\prime \prime}+\lambda z^{3} f^{\prime \prime \prime}\right)\right) /\left((1-\delta) f+\delta\left(z f^{\prime}+\lambda z^{2} f^{\prime \prime}\right)\right)\right)-(A+1)}-1 \mid \\
& -\operatorname{Re}\left[\frac{(B-1)\left(\left((1-\delta) z f^{\prime}+\delta\left(z f^{\prime}+(1+2 \lambda) z^{2} f^{\prime \prime}+\lambda z^{3} f^{\prime \prime \prime}\right)\right) /\left((1-\delta) f+\delta\left(z f^{\prime}+\lambda z^{2} f^{\prime \prime}\right)\right)\right)-(A-1)}{(B+1)\left(\left((1-\delta) z f^{\prime}+\delta\left(z f^{\prime}+(1+2 \lambda) z^{2} f^{\prime \prime}+\lambda z^{3} f^{\prime \prime \prime}\right)\right) /\left((1-\delta) f+\delta\left(z f^{\prime}+\lambda z^{2} f^{\prime \prime}\right)\right)\right)-(A+1)}-1\right] \\
& \leq(k+1)\left|\frac{(B-1)\left(\left((1-\delta) z f^{\prime}+\delta\left(z f^{\prime}+(1+2 \lambda) z^{2} f^{\prime \prime}+\lambda z^{3} f^{\prime \prime \prime}\right)\right) /\left((1-\delta) f+\delta\left(z f^{\prime}+\lambda z^{2} f^{\prime \prime}\right)\right)\right)-(A-1)}{(B+1)\left(\left((1-\delta) z f^{\prime}+\delta\left(z f^{\prime}+(1+2 \lambda) z^{2} f^{\prime \prime}+\lambda z^{3} f^{\prime \prime \prime}\right)\right) /\left((1-\delta) f+\delta\left(z f^{\prime}+\lambda z^{2} f^{\prime \prime}\right)\right)\right)-(A+1)}-1\right| \\
& =2(k+1)\left|\frac{(1-\delta)\left(f-z f^{\prime}\right)-\delta\left((1+\lambda) z^{2} f^{\prime \prime}+\lambda z^{3} f^{\prime \prime \prime}\right)}{(B+1)\left((1-\delta) z f^{\prime}+\delta\left(z f^{\prime}+(1+2 \lambda) z^{2} f^{\prime \prime}+\lambda z^{3} f^{\prime \prime \prime}\right)\right)-(A+1)\left((1-\delta) f+\delta\left(z f^{\prime}+\lambda z^{2} f^{\prime \prime}\right)\right)}\right| \\
& =2(k+1)\left|\frac{\sum_{n=2}^{\infty}(n-1) \Phi_{n} a_{n} z^{n}}{(B-A) z+\sum_{n=2}^{\infty}\left\{(B+1) \Psi_{n}-(A+1) \Phi_{n}\right\} a_{n} z^{n}}\right| \leq 2(k+1) \\
& \cdot \frac{\sum_{n=2}^{\infty} \Phi_{n}\left|a_{n}\right|}{|B-A|-\sum_{n=2}^{\infty}\left|(B+1) \Psi_{n}-(A+1) \Phi_{n}\right|\left|a_{n}\right|} .
\end{aligned}
$$


This expression is bounded above by 1 if

$$
\begin{aligned}
& \sum_{n=2}^{\infty}\{2(k+1)(n-1) \Phi(n, \delta, \lambda) \\
& \quad+|(B+1) \Psi(n, \delta, \lambda)-(A+1) \Phi(n, \delta, \lambda)|\}\left|a_{n}\right| \\
& \quad \leq|B-A|
\end{aligned}
$$

When $\delta=0$, we obtain the main result proved by Noor et at. [8] stated as follows.

Corollary 3. A function $f \in \mathscr{A}$ given by (1) is in the class $k-\mathcal{U} \mathcal{S}[A, B]$, if it satisfies the following condition:

$$
\begin{aligned}
& \sum_{n=2}^{\infty}\{2(k+1)(n-1)+|(B+1) n-(A+1)|\}\left|a_{n}\right| \\
& \quad \leq|B-A| .
\end{aligned}
$$

When $\delta=1, \lambda=0$, then we have the following known result proved by Noor et at. [8] stated as follows.

Corollary 4. A function $f \in \mathscr{A}$ given by (1) is in the class $k-\mathcal{U} \mathcal{S} \mathscr{T}[A, B]$, if it satisfies the following condition:

$$
\begin{aligned}
& \sum_{n=2}^{\infty} n\{2(k+1)(n-1)+|(B+1) n-(A+1)|\}\left|a_{n}\right| \\
& \quad \leq|B-A| .
\end{aligned}
$$

Theorem 5. A necessary and sufficient condition for any function $f(z)$ given by (2) to be in the class $k-\mathscr{V} \mathscr{U} \mathcal{S} \mathscr{T}(A, B, \delta, \lambda)$ is that

$$
\begin{aligned}
& \sum_{n=2}^{\infty}\left\{2(k+1)(n-1) \Phi_{n}+\left|(B+1) \Psi_{n}-(A+1) \Phi_{n}\right|\right\} \\
& \cdot a_{n} \leq|B-A| .
\end{aligned}
$$

Proof. In view of Theorem 2, we need only to prove the necessity. If $f \in k-\mathscr{V} \mathscr{U} \mathcal{S} \mathscr{T}(A, B, \delta, \lambda)$ and $z$ is real, then, by relation (6), we have

$$
\begin{aligned}
& \frac{(B-A)-\sum_{n=2}^{\infty}\left[(B-1) \Psi_{n}-(A-1) \Phi_{n}\right] a_{n} z^{n-1}}{(B-A)-\sum_{n=2}^{\infty}\left[(B+1) \Psi_{n}-(A+1) \Phi_{n}\right] a_{n} z^{n-1}} \\
& \geq 2 k\left|\frac{(n-1) \Phi_{n} a_{n} z^{n-1}}{(B-A)-\sum_{n=2}^{\infty}\left[(B+1) \Psi_{n}-(A+1) \Phi_{n}\right] a_{n} z^{n-1}}\right| .
\end{aligned}
$$

Letting $z \longrightarrow 1^{-}$along the real axis, we obtain the required inequality (13).

Corollary 6. Let $f(z) \in k-\mathscr{V} \mathscr{U} \mathcal{S} \mathscr{T}(A, B, \delta, \lambda)$ and be of the form (2). Then

$$
a_{n} \leq \frac{|B-A|}{2(k+1)(n-1) \Phi_{n}+\left|(B+1) \Psi_{n}-(A+1) \Phi_{n}\right|},
$$

Next, we give the growth and distortion theorem for the class $k-\mathscr{V} \mathcal{U} \mathcal{S} \mathscr{T}(A, B, \delta, \lambda)$.

Theorem 7. Let the function $f(z)$ given by (2) be in the class $k-\mathscr{V} \mathcal{U} \mathcal{S} \mathscr{T}(A, B, \delta, \lambda)$. Then, for $|z|<1$, we have

$$
|f(z)|
$$

$$
\geq r-\frac{|B-A|}{2(k+1) \Phi_{2}+\left|(B+1) \Psi_{2}-(A+1) \Phi_{2}\right|} r^{2},
$$

and

$$
\begin{aligned}
& |f(z)| \\
& \quad \leq r+\frac{|B-A|}{2(k+1) \Phi_{2}+\left|(B+1) \Psi_{2}-(A+1) \Phi_{2}\right|} r^{2} .
\end{aligned}
$$

Proof. From Theorem 5, for $n \geq 2$, we have

$$
\begin{aligned}
2(k+1) \Phi_{2}+\left|(B+1) \Psi_{2}-(A+1) \Phi_{2}\right| \sum_{n=2}^{\infty} a_{n} \\
\leq 2(k+1)(n-1) \Phi_{n} \\
\quad+\left|(B+1) \Psi_{n}-(A+1) \Phi_{n}\right| \sum_{n=2}^{\infty} a_{n} \leq|B-A| .
\end{aligned}
$$
have

For $f(z)$ given by (2) and using the triangle inequality we

$$
\begin{aligned}
& |f(z)| \geq z-\sum_{n=2}^{\infty} a_{n}|z|^{n} \geq r-r^{2} \sum_{n=2}^{\infty} a_{n} \\
& \quad \geq r-\frac{|B-A|}{2(k+1) \Phi_{2}+\left|(B+1) \Psi_{2}-(A+1) \Phi_{2}\right|} r^{2},
\end{aligned}
$$

and

$$
\begin{aligned}
& |f(z)| \leq z+\sum_{n=2}^{\infty} a_{n}|z|^{n} \leq r+\sum_{n=2}^{\infty} a_{n} r^{n}|f(z)| \\
& \quad \leq r+\frac{|B-A|}{2(k+1) \Phi_{2}+\left|(B+1) \Psi_{2}-(A+1) \Phi_{2}\right|} r^{2} .
\end{aligned}
$$

Theorem 8. Let $f(z) \in k-\mathscr{V} \mathscr{U} \mathcal{S} \mathscr{T}(A, B, \delta, \lambda)$ be of the form (1). Then, for $|z|<1$, we have

$$
\begin{aligned}
1 & -\frac{2|B-A|}{2(k+1) \Phi_{2}+\left|(B+1) \Psi_{2}-(A+1) \Phi_{2}\right|} r \\
& \leq\left|f^{\prime}(z)\right| \\
& \leq 1+\frac{2|B-A|}{2(k+1) \Phi_{2}+\left|(B+1) \Psi_{2}-(A+1) \Phi_{2}\right|} r .
\end{aligned}
$$

Proof. By differentiating (2) and after some simplification we have

$$
\left|f^{\prime}(z)\right| \geq 1-\sum_{n=2}^{\infty} n a_{n} z^{n-1} \geq 1-r \sum_{n=2}^{\infty} n a_{n}
$$


and

$$
\left|f^{\prime}(z)\right| \leq 1+\sum_{n=2}^{\infty} n a_{n} z^{n-1} \leq 1+r \sum_{n=2}^{\infty} n a_{n} .
$$

As $f \in k-\mathscr{V} \mathscr{U} \mathscr{S} \mathscr{T}(A, B, \delta, \lambda)$ so, from Theorem 5,

$$
\begin{aligned}
& \frac{2(k+1) \Phi_{2}+\left|(B+1) \Psi_{2}-(A+1) \Phi_{2}\right|}{2} \sum_{n=2}^{\infty} n a_{n} \\
& \quad \leq\left(2(k+1)(n-1) \Phi_{n}\right. \\
& \left.\quad+\left|(B+1) \Psi_{n}-(A+1) \Phi_{n}\right|\right) \sum_{n=2}^{\infty} n a_{n} \leq|B-A|,
\end{aligned}
$$

or equivalently

$$
\begin{aligned}
& \sum_{n=2}^{\infty} n\left|a_{n}\right| \\
& \quad \leq \frac{2|B-A|}{\left[2(k+1) \Phi_{2}+\left|(B+1) \Psi_{2}-(A+1) \Phi_{2}\right|\right]} .
\end{aligned}
$$

Using (25) in (22) and (23) yields required inequality (23).

Theorem 9. Let $\mu_{v} \geq 0$, for $\nu=1,2, \ldots$, l and $\sum_{\nu=1}^{l} \mu_{v} \leq 1$. If the functions $F_{\nu}(z)=z-\sum_{n=2}^{\infty} a_{n, v} z^{n}, a_{n, v} \geq 0$ are in the class $k-\mathscr{V} \mathscr{U} S \mathscr{T}(A, B, \delta, \lambda)$ then

$$
\begin{gathered}
f(z)=z-\sum_{n=2}^{\infty} A_{n} z^{n} \\
A_{n}=\sum_{\nu=1}^{l} \mu_{\nu} a_{n, v},
\end{gathered}
$$

is in class $k-\mathscr{V} \mathscr{U} \mathcal{S} \mathscr{T}(A, B, \delta, \lambda)$.

Proof. The proof follows immediately by using Theorem 5 .

Theorem 10. Let $\mu_{n} \geq 0, \sum_{n=1}^{\infty} \mu_{n}=1, f_{1}(z)=z$, and

$$
\begin{aligned}
& f_{n}(z)=z \\
& -\sum_{n=2}^{\infty} \frac{|B-A|}{\left(2(k+1)(n-1) \Phi_{n}+\left|(B+1) \Psi_{n}-(A+1) \Phi_{n}\right|\right)} z^{n}, \\
& n \geq 2 .
\end{aligned}
$$

$$
r_{1}=\inf _{n \geq 2}\left\{\frac{(1-\rho)\left(2(k+1)(n-1) \Phi_{n}+\left|(B+1) \Psi_{n}-(A+1) \Phi_{n}\right|\right)}{n|B-A|}\right\}^{1 /(n-1)} .
$$

Then $f(z)$ is in the class $k-\mathscr{V} \mathscr{U} \mathcal{S} \mathscr{T}(A, B, \delta, \lambda)$ if and only if it can be expressed in the form

$$
f(z)=\sum_{n=1}^{\infty} \mu_{n} f_{n}(z)
$$

Proof. Consider

$$
\begin{aligned}
f(z) & =\sum_{n=1}^{\infty} \mu_{n} f_{n}(z)=z \\
& -\sum_{n=2}^{\infty} \frac{|B-A|}{\left(2(k+1)(n-1) \Phi_{n}+\left|(B+1) \Psi_{n}-(A+1) \Phi_{n}\right|\right)} \mu_{n} z^{n}
\end{aligned}
$$

$n \geq 2$.

Then, by Theorem 5, we have

$$
\begin{aligned}
& \sum_{n=2}^{\infty} \frac{\left(2(k+1)(n-1) \Phi_{n}+\left|(B+1) \Psi_{n}-(A+1) \Phi_{n}\right|\right)}{|B-A|} \\
& \times \frac{|B-A|}{\left(2(k+1)(n-1) \Phi_{n}+\left|(B+1) \Psi_{n}-(A+1) \Phi_{n}\right|\right)} \\
& \cdot \mu_{n}=\sum_{n=2}^{\infty} \mu_{n}=1-\mu_{1}<1,
\end{aligned}
$$

and hence $f(z) \in k-\mathscr{V} \mathscr{U} \mathcal{S} \mathscr{T}(A, B, \delta, \lambda)$.

Conversely assume that $f(z)$ given by (2) is in the class $k-\mathscr{V} \mathscr{U} \mathcal{S} \mathscr{T}(A, B, \delta, \lambda)$. Then

$a_{n}$

$$
\begin{array}{r}
\leq \frac{|B-A|}{\left(2(k+1)(n-1) \Phi_{n}+\left|(B+1) \Psi_{n}-(A+1) \Phi_{n}\right|\right)} \\
n \geq 2 .
\end{array}
$$

By setting

$$
\begin{aligned}
& \mu_{n} \\
& =\frac{\left(2(k+1)(n-1) \Phi_{n}+\left|(B+1) \Psi_{n}-(A+1) \Phi_{n}\right|\right)}{|B-A|}
\end{aligned}
$$

$$
n \geq 2 \text {, }
$$

and $\mu_{1}=1-\sum_{n=2}^{\infty} \mu_{n}$, we can see that $f(z)$ can be expressed in the form (28), which completes the proof.

Theorem 11. Let the function $f(z)$ of the form (2) be in the class $k-\mathscr{V} \mathscr{U} \mathcal{S}(A, B, \delta, \lambda)$. Then $f(z)$ is close to convex of order $\rho(0 \leq \rho<1)$ in $|z|<r_{1}$, 
Proof. It is well-known that $f \in \mathscr{K}$ for $|z|<r_{1}$ if

$$
\left|f^{\prime}(z)-1\right| \leq 1-\rho \quad \text { for }|z|<r_{1}
$$

(see [12]), where $r_{1}$ is given by (33).

From (2), we have

$$
\left|f^{\prime}(z)-1\right| \leq \sum_{n=2}^{\infty} n a_{n} z^{n-1}
$$

Clearly $\left|f^{\prime}(z)-1\right| \leq 1-\rho$, if

$$
\sum_{n=2}^{\infty} \frac{n}{1-\rho}|z|^{n-1} \leq 1
$$

As $f \in k-\mathscr{V} \mathscr{U} \mathcal{S} \mathscr{T}(A, B, \delta, \lambda)$, from Theorem 5, inequality (36) will be true if

$$
\begin{aligned}
& \frac{n}{1-\rho}|z|^{n-1} \\
& \quad \leq \frac{2(k+1)(n-1) \Phi_{n}+\left|(B+1) \Psi_{n}-(A+1) \Phi_{n}\right|}{|B-A|},
\end{aligned}
$$

$$
|z| \leq\left\{\frac{(1-\rho)\left(2(k+1)(n-1) \Phi_{n}+\left|(B+1) \Psi_{n}-(A+1) \Phi_{n}\right|\right)}{n|B-A|}\right\}^{1 /(n-1)}
$$

Theorem 12. Let $f(z)$ of form (2) be in the class $k$ $\mathscr{V} \mathcal{U} \mathcal{S} \mathscr{T}(A, B, \delta, \lambda)$. Then $f(z)$ is starlike of order $\rho(0 \leq \rho<$ 1) in $|z|<r_{2}$,

$$
r_{2}=\left\{\frac{(1-\rho)\left(2(k+1)(n-1) \Phi_{n}+\left|(B+1) \Psi_{n}-(A+1) \Phi_{n}\right|\right)}{(n-\rho)|B-A|}\right\}^{1 /(n-1)} .
$$

Proof. To prove $f \in \mathcal{S} \mathscr{T}(\rho)$, for $|z|<r_{2}$ it is sufficient to show

$$
\left|\frac{z f^{\prime}(z)}{f(z)}-1\right| \leq 1-\rho \quad \text { for }|z|<r_{2}
$$

(see [12]). From (2) we have

$$
\left|\frac{z f^{\prime}(z)}{f(z)}-1\right| \leq \frac{\sum_{n=2}^{\infty}(n-1) a_{n}|z|^{n-1}}{1-\sum_{n=2}^{\infty} a_{n}|z|^{n-1}}
$$

Thus $\left|z f^{\prime}(z) / f(z)-1\right| \leq 1-\rho$, if

$$
\sum_{n=2}^{\infty} \frac{(n-\rho)}{1-\rho} a_{n}|z|^{n-1} \leq 1
$$

As $f \in k-\mathscr{V} \mathscr{U} \mathcal{S} \mathscr{T}(A, B, \delta, \lambda)$, (42) must hold if

$$
\begin{aligned}
& \frac{(n-\rho)}{1-\rho}|z|^{n-1} \\
& \leq \frac{\left(2(k+1)(n-1) \Phi_{n}+\left|(B+1) \Psi_{n}-(A+1) \Phi_{n}\right|\right)}{|B-A|},
\end{aligned}
$$

which gives us $|z|<r_{2}$, where $r_{2}$ is given by (39).
Next we prove that the class $k-\mathscr{V} \mathscr{U} \mathcal{S} \mathscr{T}(A, B, \delta, \lambda)$ is closed under generalized Bernardi-Livingston operator defined as

$$
L_{c}(f)=\frac{c+1}{z^{c}} \int_{0}^{z} t^{c-1} f(t) d t, \quad c>-1
$$

(see [17]).

Theorem 13. If $f(z) \in k-\mathscr{V} \mathscr{U} \mathcal{S} \mathscr{T}(A, B, \delta, \lambda)$ then $L_{c}(f) \in$ $k-\mathscr{V} \mathcal{U} \mathcal{S} \mathscr{T}(A, B, \delta, \lambda)$.

Proof. Consider

$$
L_{c}(f)=\frac{c+1}{z^{c}} \int_{0}^{z} t^{c-1} f(t) d t
$$

using (2) and, after integration, we obtain

$$
L_{c}(f)=z-\sum_{n=2}^{\infty} \frac{c+1}{c+n} a_{n} z^{n} .
$$

Since $f(z) \in k-\mathscr{V} \mathcal{U} \mathcal{S}(A, B, \delta, \lambda)$, so an easy calculation leads to 


$$
\begin{aligned}
& \sum_{n=2}^{\infty}\left\{2(k+1)(n-1) \Phi_{n}+\left|(B+1) \Psi_{n}-(A+1) \Phi_{n}\right|\right\} \\
& \cdot\left(\frac{c+1}{c+n}\right) a_{n} \leq \sum_{n=2}^{\infty}\left(2(k+1)(n-1) \Phi_{n}\right. \\
& \left.\quad+\left|(B+1) \Psi_{n}-(A+1) \Phi_{n}\right|\right) a_{n} \leq|B-A|
\end{aligned}
$$

and, therefore, $L_{c}(f) \in k-\mathscr{V} \mathscr{U} \mathcal{S} \mathscr{T}(A, B, \delta, \lambda)$.

\section{Data Availability}

There is no data for this article.

\section{Conflicts of Interest}

The authors declare that they have no conflicts of interest.

\section{Authors' Contributions}

All authors jointly work on results, and they read and approved the final manuscript.

\section{Acknowledgments}

The work here is supported by UKM grant: GUP-2017-064 and DPP-2015-FST.

\section{References}

[1] H. Silverman, "Univalent functions with negative coefficients," Proceedings of the American Mathematical Society, vol. 51, pp. 109-116, 1975.

[2] A. W. Goodman, “On uniformly convex functions," Annales Polonici Mathematici, vol. 56, no. 1, pp. 87-92, 1991.

[3] A. W. Goodman, "On uniformly starlike functions," Journal of Mathematical Analysis and Applications, vol. 155, no. 2, pp. 364370, 1991.

[4] W. C. Ma and D. Minda, "Uniformly convex functions," Annales Polonici Mathematici, vol. 57, no. 2, pp. 165-175, 1992.

[5] F. Ronning, "Uniformly convex functions and corresponding class of starlike functions," Proceedings of the American Mathematical Society, vol. 118, no. 1, pp. 189-196, 1993.

[6] J. Sokol and A. Wisniowska-Wajnryb, "On some classes of starlike functions related with parabola," Folia Sci. Univ. Tech. Resov, vol. 121, no. 18, pp. 35-42, 1993.

[7] J. Sokol and A. Wisniowska-Wajnryb, "On certain problem in the classes of k-starlike functions," Computers \& Mathematics with Applications, vol. 62, no. 12, pp. 4733-4741, 2011.

[8] K. I. Noor and S. N. Malik, "On coefficient inequalities of functions associated with conic domains," Computers \& Mathematics with Applications, vol. 62, pp. 2209-2217, 2011.

[9] R. M. Ali, S. R. Mondal, and V. Ravichandran, "On the Janowski convexity and starlikeness of the confluent hypergeometric function," Bulletin of the Belgian Mathematical Society - Simon Stevin, vol. 22, no. 2, pp. 227-250, 2015.

[10] R. M. Ali and V. Ravichandran, "Uniformly convex and uniformly starlike functions," Mathematics Newsletter, vol. 21, pp. 16-30, 2011.
[11] M. K. Aouf, H. M. Hossen, and A. Y. Lashin, "On certain families of analytic functions with negative coefficients," Indian Journal of Pure and Applied Mathematics, vol. 31, no. 8, pp. 9991015, 2000.

[12] M. K. Aouf, A. A. Shamandy, A. O. Mostafa, and A. K. Wagdy, "Certain subclasses of uniformly starlike and convex functions defined by convolution with negative coefficients," Matematicki Vesnik, vol. 65, no. 1, pp. 14-28, 2013.

[13] S. Kanas and H. M. Srivastava, "Linear operators associated with $k$-uniformly convex functions," Integral Transforms and Special Function, vol. 9, no. 2, pp. 121-132, 2000.

[14] S. A. Kanas and A. Wisniowska, "Conic domains and starlike functions," Revue Roumaine de Mathématique Pures et Appliquées, vol. 45, no. 4, pp. 647-657, 2000.

[15] N. Magesh, "Certain subclasses of uniformly convex functions of order $\alpha$ and type $\beta$ with varying arguments," Journal of the Egyptian Mathematical Society, vol. 21, no. 3, pp. 184-189, 2013.

[16] R. Bharati, R. Parvatham, and A. Swaminathan, "On subclasses of uniformly convex functions and corresponding class of starlike functions," Tamkang Journal of Mathematics, vol. 28, no. 1, pp. 17-32, 1997.

[17] S. D. Bernardi, "Convex and starlike univalent functions," Transactions of the American Mathematical Society, vol. 135, pp. 429-446, 1969. 


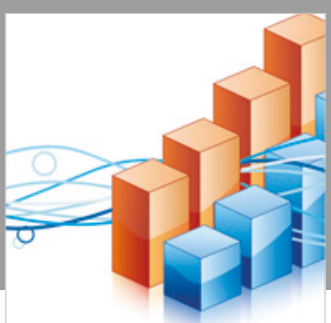

Advances in

Operations Research

\section{-n-m}
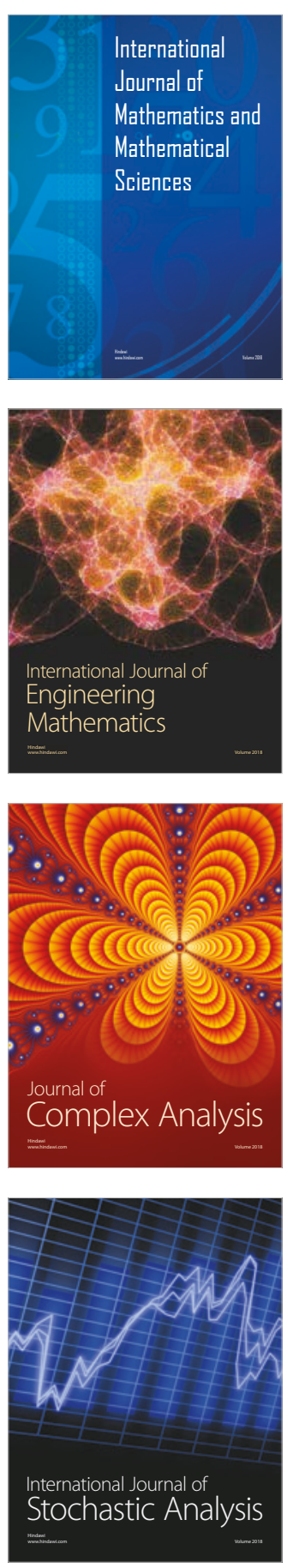
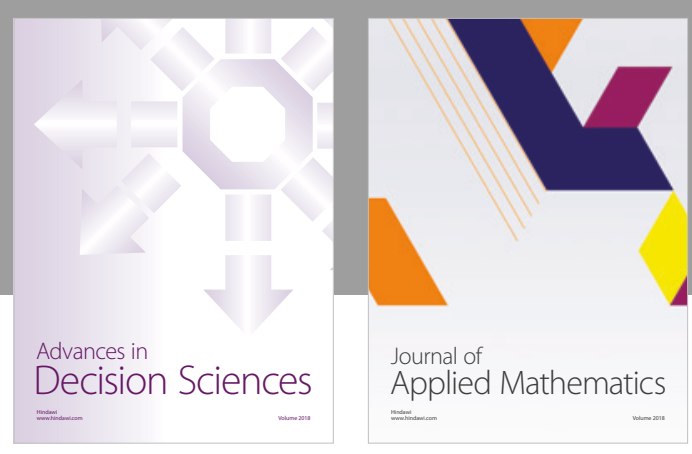

Journal of

Applied Mathematics
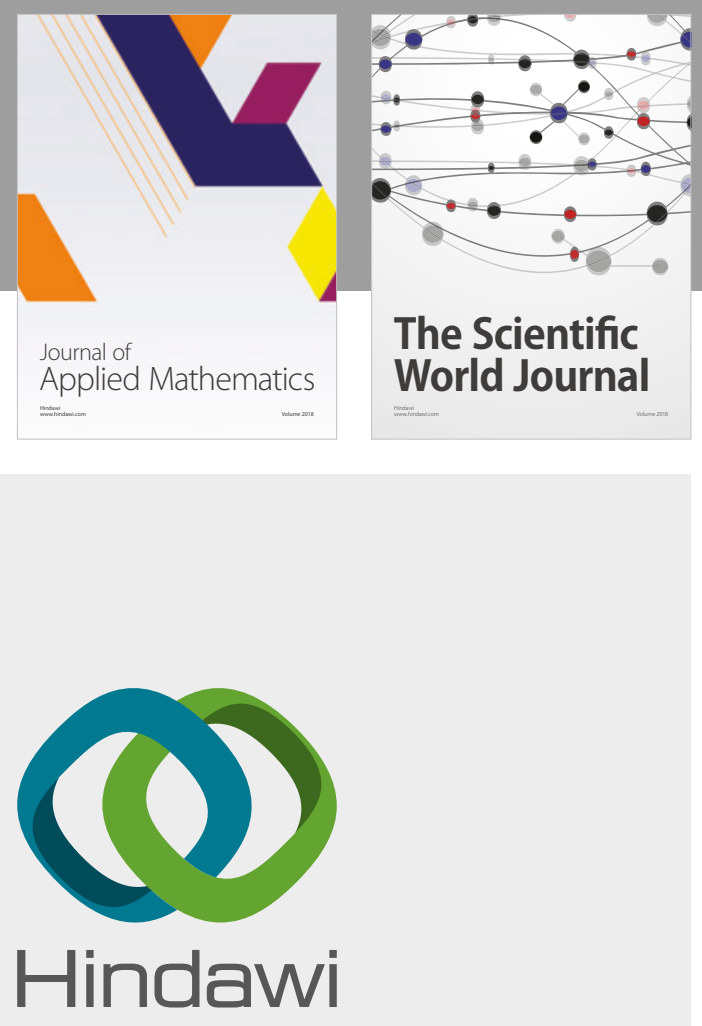

Submit your manuscripts at

www.hindawi.com

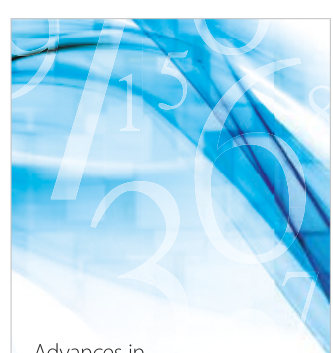

Advances in
Numerical Analysis
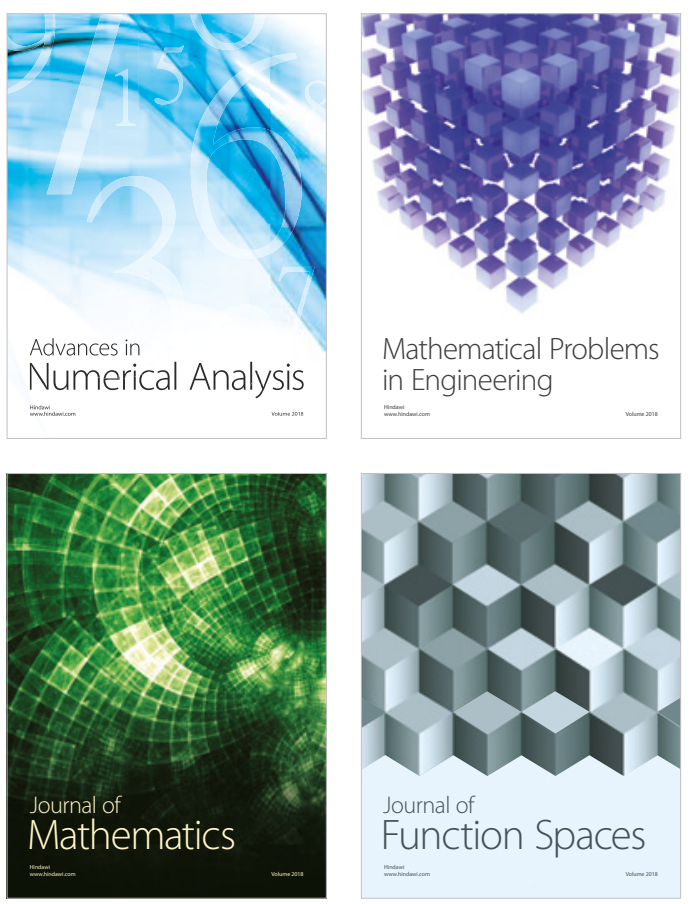

Mathematical Problems in Engineering

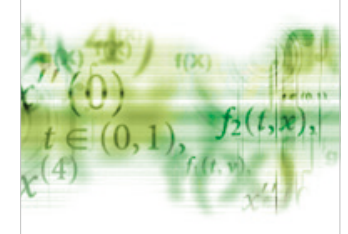

International Journal of

Differential Equations

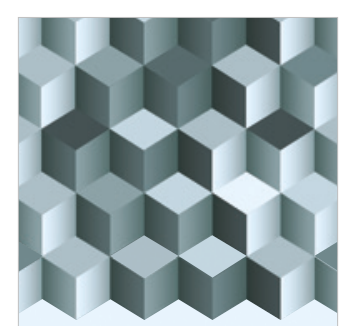

Journal of

Function Spaces

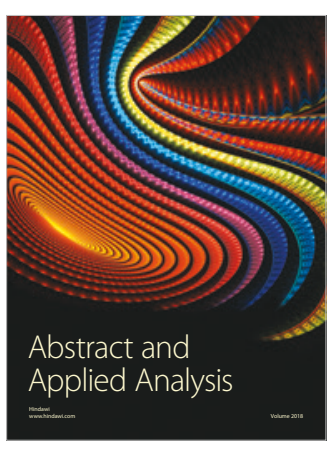

The Scientific

World Journal

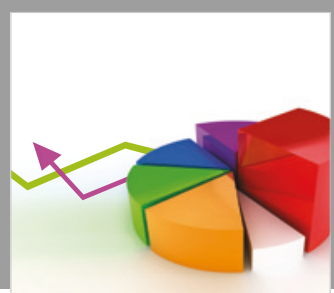

Journal of

Probability and Statistics
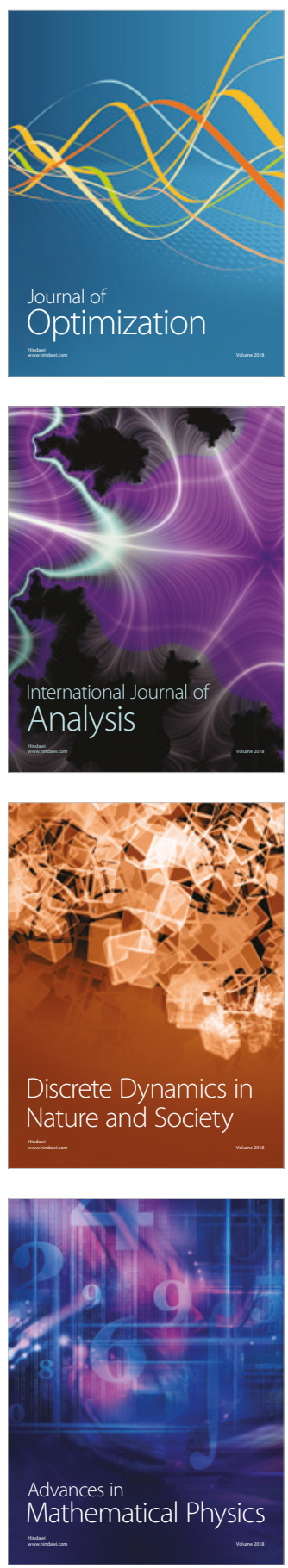